\title{
Design, molecular docking, drug-likeness, and molecular dynamics studies of 1,2,4-trioxane derivatives as novel Plasmodium falciparum falcipain-2 (FP-2) inhibitors
}

\author{
Subham Ghosh ${ }^{1}$, Dipak Chetia ${ }^{1}$, NeElutPal Gogoi ${ }^{1}$, Mithun RUdRAPaL ${ }^{2}$ * \\ ${ }^{1}$ Deptartment of Pharmaceutical Sciences, Dibrugarh University, Dibrugarh, Assam, India \\ ${ }^{2}$ Department of Pharmaceutical Chemistry, Rasiklal M. Dhariwal Institute of Pharmaceutical Education and Research, \\ Chinchwad, Pune, Maharashtra, India
}

\begin{abstract}
Despite significant progress made in drug discovery and development over the past few decades, malaria remains a life-threatening infectious disease across the globe. Because of the widespread emergence of drug-resistant strains of Plasmodium falciparum, the clinical utility of existing drug therapies including Artemisinin-based Combination Therapies (ACTs) in the treatment of malaria has been increasingly limited. It has become a serious health concern which, therefore, necessitates the development of novel drug molecules and/or alternative therapies to combat, particularly resistant $P$. falciparum. The objective of the present study was to develop 1,2,4-trioxane derivatives as novel antimalarial agents that would be effective against resistant $P$. falciparum. In our study, 15 new trioxane derivatives were designed by molecular modification of the 1,2,4-trioxane scaffold as possible antimalarial agents. Molecular modeling studies of trioxane derivatives were performed based on the CADD approach using Biovia Discovery Studio (DS) 2018 software. The protein-ligand docking study was performed against $P$. falciparum falcipain 2 (FP-2) using the simulation-based docking protocol LibDock by the flexible docking method. The assessment of drug-likeness, ADMET properties, and toxicity was also performed. Furthermore, the compounds $\mathrm{CC} 3$ and $\mathrm{CC} 7$, which showed the best binding affinity against the target $P$. falciparum $\mathrm{FP}-2$, were investigated by molecular dynamics (MD) simulation studies followed by the calculation of MM-PBSA binding free energy of protein-ligand complexes using DS 2020. Results of the docking study showed that among the 15 compounds, three trioxane derivatives were found to possess promising binding affinity with LibDock scores ranging from 117.16 to 116.90 . Drug-likeness, ADMET, and toxicity properties were found to be satisfactory for all the compounds. Among the 15 compounds, two compounds, namely $\mathrm{CC} 3$ and $\mathrm{CC} 7$, showed the highest binding affinity against FP-2 with LibDock score of 117.166 and 117.200, respectively. The Libdock score of the co-crystal inhibitor was 114.474. MD studies along with MM-PBSA calculations of binding energies further confirmed the antimalarial potential of the compounds $\mathrm{CC} 3$ and $\mathrm{CC} 7$, with the formation of well-defined and stable receptor-ligand interactions against the $P$. falciparum FP-2 enzyme. Additionally, the selectivity of trioxane hits identified as potential inhibitors of $P$. falciparum cysteine protease FP-2 was determined on human cysteine proteases such as cathepsins (Cat K and Cat L), which are host homologous. Finally, it was concluded that the newly designed 1,2,4trioxane derivatives can be further studied for in vitro and in vivo antimalarial activities for their possible development as potent antimalarial agents effective against resistant $P$. falciparum.
\end{abstract}

Key words: 1,2,4-trioxane, Plasmodium falciparum, drug resistance, molecular docking, molecular dynamics, falcipain 2 inhibitors

Abbreviations
ACTs

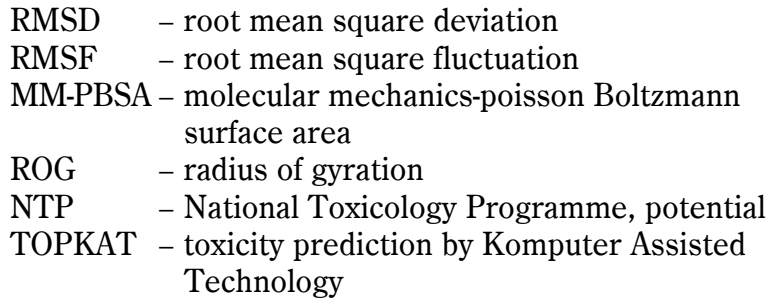
Technology

\footnotetext{
* Corresponding author: Deptartment of Pharmaceutical Chemistry, Rasiklal M. Dhariwal Institute of Pharmaceutical Education and Research, Chinchwad, Pune-411019, Maharashtra, India; e-mail: rsmrpal@gmail.com
} 


\section{Introduction}

Malaria remains one of the most devastating infectious diseases affecting millions of people every year across the globe (Kalita et al., 2020). Plasmodium falciparum, the deadliest malarial parasite, is responsible for most of the mortality associated with malaria infection (Kalita et al., 2019; Rudrapal et al., 2018). Artemisininbased Combination Therapies (ACTs) are currently the frontline treatments against $P$. falciparum malaria (Patowary et al., 2019; Medhi et al., 2018; Gogoi et al., 2016; Rudrapal et al., 2016a). Despite the significant progress made in drug discovery and development over the past few decades, the control and prevention of malaria are still a challenging task. Because of the widespread emergence of drug-resistant strains of $P$. falciparum, the clinical utility of existing drug therapies including ACTs in the treatment of malaria has been increasingly limited (Roy et al., 2013; Rudrapal et al., 2013; Cumming et al., 1998; Neil et al., 2005). It has become a serious health concern which, therefore, necessitates the development of novel drug molecules and/or alternative therapies to combat, particularly resistant $P$. falciparum malaria.

Artemisinin (ART) is a naturally occurring antimalarial lead molecule effective against $P$. falciparum malaria. The 1,2,4-trioxane moiety is believed to be the key pharmacophore of ART and its semi-synthetic derivatives (Posner, 1997; Chakroborty et al., 2012; Opensica et al., 2009; Rudrapal et al., 2019). ART exhibits antimalarial action by forming highly alkylating free radicals and reactive oxygen species after interaction with iron (Rudrapal et al., 2017; Chetia et al., 2016). The 1,2,4-trioxane moiety containing the peroxide functional group is cleaved during the erythrocytic stage of parasite growth, leading to the release of reactive oxygen radicals that kill the parasite by peroxidation of parasitic proteins or cell membrane (O'Neill et al., 2005; Wang et al., 2009; Robert et al., 2020; Biamonte et al., 2013; Mugumbate et al., 2013). The objective of the present study was to develop 1,2,4-trioxane derivatives as novel antimalarial agents that would be effective against resistant $P$. falciparum. In our study, 15 new trioxane derivatives were designed as possible antimalarial agents by molecular modification of the 1,2,4-trioxane scaffold. Molecular modeling studies of trioxane derivatives were performed based on the CADD approach using Biovia Discovery Studio (DS) 2018 software. The protein-ligand docking study was conducted against $P$. falciparum cysteine protease FP-2 (PDB ID: 3BPF) protein using the simulationbased docking protocol LibDock by the flexible docking method. The assessment of drug-likeness, ADMET properties, and toxicity was also performed. Further, molecular dynamics (MD) simulation studies were performed along with the calculation of MM-PBSA-based free binding energies of docked complexes to confirm the antimalarial potential of the best scored compounds against the $P$. falciparum FP-2 enzyme. Additionally, the selectivity of trioxane hits identified as potential inhibitors of $P$. falciparum cysteine protease FP-2 was determined on human cysteine proteases such as cathepsins (Cat K and Cat $\mathrm{L}$ ), which are the host homologous proteins.

\section{Materials and methods}

\section{Design of new 1,2,4-trioxane derivatives and molecular modeling studies}

In this study, a 1,2,4-trioxane scaffold was used as the basic structural framework for designing new trioxane derivatives by the molecular modification approach. Fifteen trioxane derivatives were designed by the substitution of the aryl moiety (substituted biphenyl-3-carbaldehyde) at $\mathrm{C} 3$ and 4-fluorobenzoyl moiety at $\mathrm{C} 6$ as depicted in Fig. 1. The trioxane ring with a bulk aryl substituent containing an aldehydic group attached at the $3^{\text {rd }}$ position could serve as an important factor for their antimalarial efficacy.

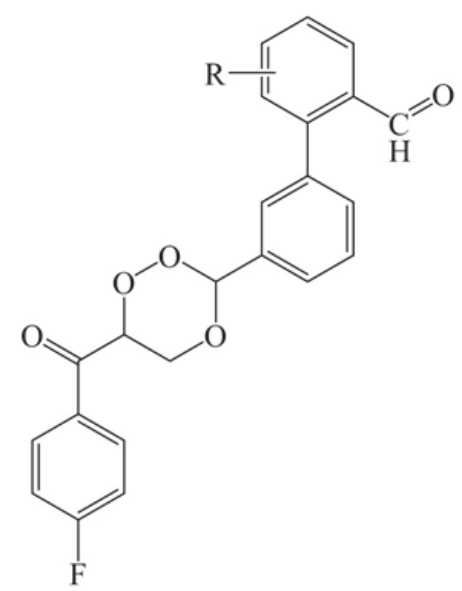

Fig. 1. Structures of the designed 1,2,4-trioxane derivatives

Molecular modeling studies were performed on Biovia Discovery Studio (DS) 2020 software (Dassault Systèmes BIOVIA, San Diego, USA) by using Dell Preci- 
sion workstation T3400 running on Intel Core 2 Duo Processor, 4 GB RAM, 250 GB hard disk, and NVidia Quodro FX 4500 graphics card.

\section{Molecular docking study}

Prior to docking, ligands and proteins were prepared according to the standard protocol of DS software. The three-dimensional structures of compounds were generated, and their energy minimization was performed using the CHARMM (Chemistry at Harvard Macromolecular Mechanics)-based smart minimizer. It performs 2000 steps of Steepest Descent followed by the Conjugate Gradient algorithm with an RMSD gradient of $0.01 \mathrm{kcal} / \mathrm{mol}$ (Mugumbate et al., 2013). The X-ray crystal structure of FP-2 (PDB ID: 3BPF) protein was retrieved from the RCSB Protein Data Bank (http:// www.rcsb.org/). Chain A of 3BPF determined at the resolution of $3.2 \AA$ was used in the study (Ghorab et al., 2020; Abdel-Hamid et al., 2014). The protein molecules were prepared by necessary cleaning, removal of water molecules, and energy minimization following the standard protocol. The energy minimization was performed using the CHARMM-based smart minimizer method at the maximum steps of 200 with RMSD gradient of $0.1 \mathrm{kcal} / \mathrm{mol}$ (Jain et al., 2008).

A receptor grid was generated around the binding cavity (active sites) of the energy-minimized protein molecule by specifying the key amino acid residues. The co-crystal ligand (active inhibitor) of the respective protein molecule was selected to define the amino acid residues for predicting the binding sites. For the generated receptor grid boxes, the binding site sphere was set with the radius of $2.90 \AA$ and $\mathrm{x}, \mathrm{y}, \mathrm{z}$ dimensions of 10.858743 , 12.558267, 85.699954 for 3BPF (Ponnan et al., 2013; Rudrapal et al., 2017).

The docking was performed using a simulation-based docking protocol, i.e., LibDock of DS 2020. LibDock is a high-throughput docking algorithm used to dock compounds into the active site(s) of a receptor molecule. All docking and consequent scoring parameters used were kept at their default settings (Kashyap et al., 2016; Sharma et al., 2016). LibDock docked compounds were estimated. All docked poses were scored and ranked. The binding affinities of docked compounds were predicted by analyzing interactions of receptor-ligand complexes. The binding modes of the best docked pose were analyzed using the $3 \mathrm{D}$ receptor-ligand complex. Different nonbonding interactions (hydrogen bonding, hydrophobic, etc.) were also analyzed using the $2 \mathrm{D}$ diagram of receptor-ligand complexes. The docking results of test compounds were compared with those of the co-crystal ligands. The docking energies of the co-crystal ligands were used as reference ligands to evaluate the binding affinity of the test compounds.

\section{Drug-likeness studies}

The molecular properties and drug-likeness parameters of the compounds $\mathrm{CC} 1-\mathrm{CC} 15$ were calculated based on theoretical approaches using DS 2020. Molecular properties such as log of octanol/water partition coefficient $(\log P)$, molecular weight $(\mathrm{MW})$, number of hydrogen bond acceptors (nHBAs), number of hydrogen bond donors (nHBDs), and molecular polar surface area (PSA) incorporated in Lipinski's rule of five (Rudrapal et al., 2019) and other physicochemical parameters such as number of aromatic rings (nARs), number of rings (nRs), and number of rotable bonds (nRotBs) were also calculated. The relatively higher lipophilicity of the target compounds could account for the good antimicrobial activities due to the increased intracellular concentration. The Molinspiration online software (http:/www. molinspiration.com/, 2016) was used to determine nonviolation of drug-likeness and drug-likeness score.

\section{ADMET prediction}

The ADME-Toxicity (ADMET) parameters were calculated using the ADMET descriptor protocol of DS 2020 software. Six mathematical models (aqueous solubility, blood-brain barrier penetration, cytochrome $\mathrm{P}_{450}$ (CYP) 2D6 inhibition, hepatotoxicity, intestinal absorption, and plasma protein binding) were used for quantitative prediction of properties related to ADMET characteristics or pharmacokinetics (PKs) of drug molecules (Rudrapal et al., 2019).

\section{Toxicity assessment}

In silico toxicities were assessed using the TOPKAT module of DS 2020 software (Rudrapal et al., 2019). The following toxicity parameters were calculated to assess carcinogenicity and mutagenicity: mouse female NTP, mouse male NTP, rat female NTP, rat male NTP, rat oral $\mathrm{LD}_{50}$, mouse female FDA, mouse male FDA, rat female FDA, rat male FDA, Ames prediction, and DTP prediction . 
Table 1. Designed 1,2,4-trioxane derivatives CC1-CC15

\begin{tabular}{c|c}
\hline $\begin{array}{c}\text { Compounds } \\
\text { code }\end{array}$ & $\mathrm{R}_{1}$ \\
\hline $\mathrm{CC} 1$ & $\mathrm{H}$ \\
\hline $\mathrm{CC} 2$ & $4-\mathrm{F}$ \\
\hline $\mathrm{CC} 3$ & $4-\mathrm{Br}$ \\
\hline $\mathrm{CC} 4$ & $4-\mathrm{CH}_{3}$ \\
\hline $\mathrm{CC} 5$ & $4-\mathrm{OH}$ \\
\hline $\mathrm{CC} 6$ & $4-\mathrm{Cl}$ \\
\hline $\mathrm{CC} 7$ & $4-\mathrm{NO}_{2}$ \\
\hline $\mathrm{CC} 8$ & $3-\mathrm{OH}$ \\
\hline $\mathrm{CC} 9$ & $3-\mathrm{CH}_{3}$ \\
\hline $\mathrm{CC} 10$ & $3-\mathrm{Cl}$ \\
\hline $\mathrm{CC} 11$ & $3-\mathrm{Br}$ \\
\hline $\mathrm{CC} 12$ & $3-\mathrm{NO}{ }_{2}$ \\
\hline $\mathrm{CC} 13$ & $2-\mathrm{Cl}$ \\
\hline $\mathrm{CC} 14$ & $2-\mathrm{CH}_{3}$ \\
\hline $\mathrm{CC} 15$ & $2-\mathrm{Br}$ \\
\hline
\end{tabular}

CC - compound code (refer Fig. 1)

\section{MD simulation}

The compounds $\mathrm{CC} 3$ and $\mathrm{CC} 7$ that showed the best binding affinities against the target protein $P$. falciparum FP-2 were further considered for the MD simulation study by using DS 2020 . The protein-ligand complexes generated from the binding affinity prediction analysis were subjected to the MD simulation study along with the original crystal structure of the target proteins complexed with the co-crystal inhibitors. The protein-ligand complexes were initially cleaned and prepared using the macromolecule tool of DS 2020 (Lin et al., 2011). The complexes were solvated using the explicit periodical boundary condition in a cubic box of water with a distance of $5 \AA$ from the boundary. The system was neutralized by adding $0.15 \mathrm{M} \mathrm{NaCl}$ during the solvation process. The solvated systems were energy minimized (5000 steps steepest descent and 5000 steps conjugate gradient with energy RMSD gradient $0.01 \mathrm{kcal} / \mathrm{mol}$ ), heated (20 ps), and equilibrated (500 ps) using the “Standard Dynamic Cascade" protocol of DS 2020. Subsequently, 30 ns production was run in NVT ensemble at $300 \mathrm{~K}$ for the whole protein-ligand complexes, where snapshots were saved at every 2 ps. For the electrostatic calculations, the Particle Mesh Ewald (PME) method and to constrain bonds containing hydrogen the SHAKE algorithm were used with the time step of $2 \mathrm{fs}$. After completing the simulation, RMSD (root mean square deviation), RMSF (root mean square fluctuation), and ROG (radius of gyration) were computed by taking the starting structure as a reference to evaluate the conformational changes of the protein-ligand complexes. Throughout the simulation period, the distances of different hydrogen bonds formed were also monitored and analyzed. Finally, different nonbond interactions were also analyzed from the average interaction of the protein-ligand complexes and compared with the interactions obtained from the starting structures (Noha et al., 2017; Lai et al., 2011).

Molecular Mechanics-Poisson Boltzmann Surface Area (MM-PBSA)-based binding free energy calculation

After MD simulation, the binding free energies for each protein-ligand complex were calculated using "Binding Free Energy-Single Trajectory" protocol of DS 2020 with the application of the MM-PBSA method. In the analysis, the binding free energies of all the generated conformations were calculated, and finally, the average binding free energy $(\Delta G)$ was determined for each protein-ligand complex.

\section{Docking for selectivity study}

To determine the selectivity of best hits for 1,2,4-trioxanes identified as potential inhibitors of $P$. falciparum cysteine protease FP-2 against human cysteine proteases, protein-ligand docking was performed with the identified trioxanes $\mathrm{CC} 3$ and $\mathrm{CC} 7$ against human homologous cysteine proteases such as cathepsins (Cat K and Cat L). The X-ray crystal structures of Cat $\mathrm{K}$ and Cat L (PDB IDs: 3OVZ and 3OF9, respectively) proteins were retrieved from the RCSB Protein Data Bank. The proteins were obtained at the resolution of $2.02 \AA$ and $1.76 \AA$, respectively. A previously reported method was used in the docking study. The simulation-based docking protocol LibDock was used to perform the docking study using the DS 2020 software. All parameters for grid generation and docking analysis were kept at the default settings of the software. 


\section{Results}

\section{Docking study}

The protein model (3BPF/E64) was validated and used for the docking study. Prior to docking, the receptor grid models were generated and optimized in terms of binding site spheres to achieve predictive interactions between receptor molecules and the test compounds (Fig. 2). The reference co-crystal ligand (E64) was successfully re-docked to the predicted active sites of protein molecule (3BPF) with an acceptable RMSD value of $3.529 \AA$. The validation study was performed to reproduce the results of ligand binding modes observed experimentally in protein-ligand complexes (Kalita et al., 2019). Results of the validation study confirmed experimental binding modes/conformations of the co-crystal inhibitor E64 in the binding pocket of the respective protein molecule $3 \mathrm{BPF}$ with predictable protein-ligand interactions (Fig. 3).

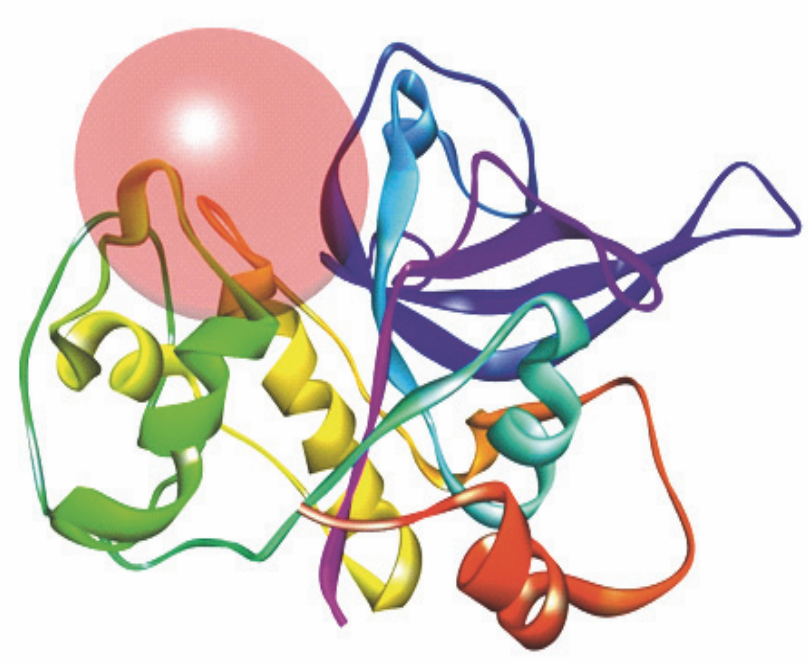

Fig. 2. Receptor grid model of $P$. falciparum FP-2 (3BPF); the figure indicates the generation of binding site/active site sphere/space in/around the FP-2 protein molecule for protein-ligand docking

All the 15 compounds (Fig. 1 ) showed almost similar binding affinities with some degree of variations. Against P. falciparum FP-2 (3BPF), compounds CC3, CC5, CC7, and $\mathrm{CC} 8$ exhibited comparatively better inhibitory activity than the remaining analogues. Results of molecular docking are summarized in Table 2.

Among the 15 compounds, the compounds $\mathrm{CC} 3$ and $\mathrm{CC} 7$ showed the highest binding affinity against FP-2

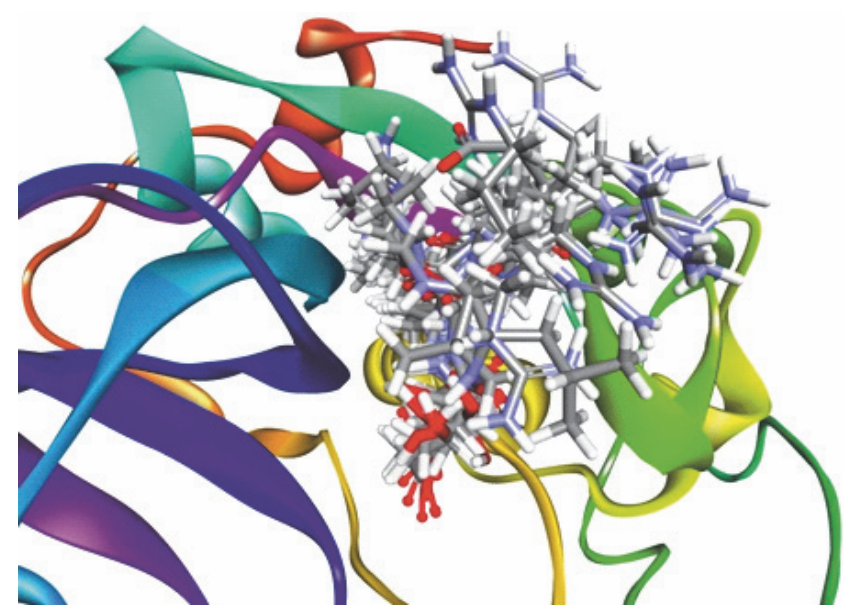

Fig. 3. Re-docked conformer of $P$. falciparum FP-2 (3BPF); the figure indicates re-docking of the co-crystal ligand E64 into the predicted binding pocket of FP-2; predictable binding modes were observed experimentally in the active sites of FP-2 for all the test 1,2,4-trioxane derivatives; all the test compounds and redocked E64 are superimposed into the predicted binding sites of the original co-crystal structure (E64) in the FP-2 protein

Table 2. LibDock scores of the designed 1,2,4-trioxane derivatives $\mathrm{CC} 1-\mathrm{CC} 15$ against $P$. falciparum FP-2 (3BPF)

\begin{tabular}{c|c|c|c}
\hline \multirow{2}{*}{$\begin{array}{c}\text { Compounds } \\
\text { code }\end{array}$} & $\begin{array}{c}\text { LibDock score } \\
\text { (3BPF) }\end{array}$ & \multicolumn{2}{|c}{ Number of bonds } \\
\cline { 3 - 4 } CC1 & 104.893 & 3 & 3 \\
\hline CC2 & 105.244 & 5 & 6 \\
\hline CC3 & 117.166 & 1 & 7 \\
\hline CC4 & 107.727 & 4 & 1 \\
\hline CC5 & 116.169 & 4 & 10 \\
\hline CC6 & 107.597 & 3 & 5 \\
\hline CC7 & 117.200 & 1 & 5 \\
\hline CC8 & 116.900 & 4 & 6 \\
\hline CC9 & 113.527 & 5 & 6 \\
\hline CC10 & 107.704 & 2 & 2 \\
\hline CC11 & 105.352 & 2 & 7 \\
\hline CC12 & 105.029 & 2 & 4 \\
\hline CC13 & 106.599 & 2 & 3 \\
\hline CC14 & 107.311 & 7 & 4 \\
\hline CC15 & 109.060 & 2 & 4 \\
\hline E64 & 114.474 & 2 & 11 \\
\hline
\end{tabular}

3BPF - P. falciparum falcipain 2 (Pf FP-2); co-crystal inhibitor - E64

with the LibDock score of 117.166 and 117.200 , respectively. The Libdock score of the co-crystal inhibitor was 114.474. These two compounds were found to ex- 


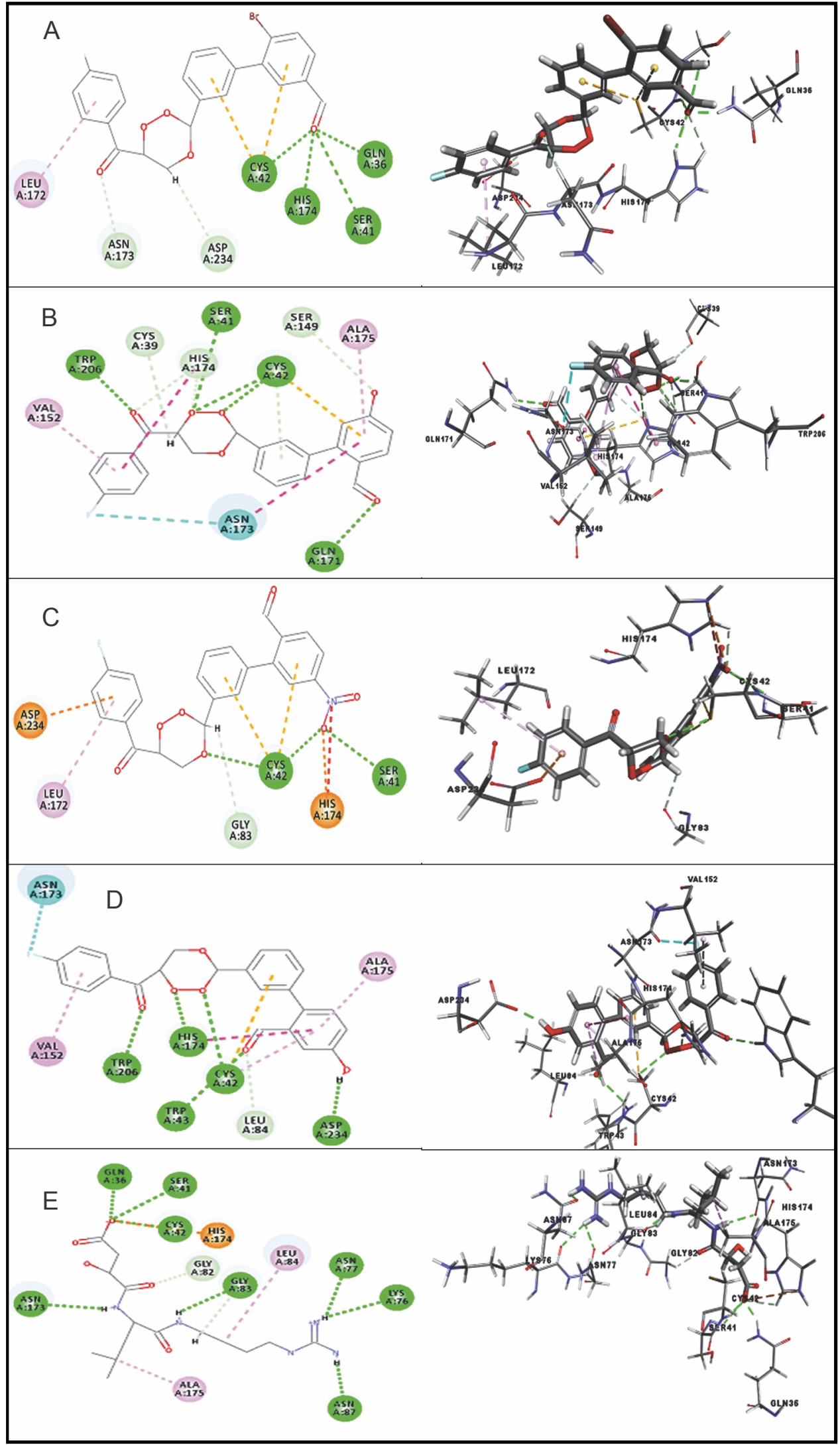

Fig. 4. Binding modes and docking interactions diagrams of compounds CC3 (A), CC5 (B), CC7 (C), CC8 (D), and the co-crystal inhibitor E64 (E) (left: 2D interaction, right: 3D interaction) against $P$. falciparum FP2 (3BPF); dotted green lines indicate conventional H-bonding interactions, and dotted yellow and purple lines indicate different types of hydrophobic interactions 
Table 3. Calculated molecular properties and drug-likeness parameters of the designed 1,2,4-trioxane derivatives CC1-CC15

\begin{tabular}{c|c|c|c|c|c|c|c|c|c}
\hline \multirow{2}{*}{$\begin{array}{c}\text { Compounds } \\
\text { code }\end{array}$} & \multicolumn{7}{|c|}{ Molecular properties and Lipinski's parameters } & \multirow{2}{*}{ DL score } \\
\cline { 2 - 9 } CC1 & ALogP & MW & nAR & nHBA & nHBD & nR & nRB & Mol. PSA & \\
\hline CC2 & 4.241 & 392.376 & 3 & 5 & 0 & 4 & 5 & 61.83 & 0.55 \\
\hline CC3 & 4.990 & 471.273 & 3 & 5 & 0 & 4 & 5 & 61.83 & 0.55 \\
\hline CC4 & 4.728 & 406.403 & 3 & 5 & 0 & 4 & 5 & 61.83 & 0.55 \\
\hline CC5 & 3.999 & 408.376 & 3 & 6 & 1 & 4 & 5 & 82.06 & 0.55 \\
\hline CC6 & 4.906 & 426.822 & 3 & 5 & 0 & 4 & 5 & 61.83 & 0.55 \\
\hline CC7 & 4.136 & 437.374 & 3 & 7 & 0 & 4 & 6 & 107.65 & 0.55 \\
\hline CC8 & 3.999 & 408.376 & 3 & 6 & 1 & 4 & 5 & 82.06 & 0.55 \\
\hline CC9 & 4.728 & 406.403 & 3 & 5 & 0 & 4 & 5 & 61.83 & 0.55 \\
\hline CC10 & 4.906 & 426.822 & 3 & 5 & 0 & 4 & 5 & 61.83 & 0.55 \\
\hline CC11 & 4.990 & 471.273 & 3 & 5 & 0 & 4 & 5 & 61.83 & 0.55 \\
\hline CC12 & 4.136 & 437.374 & 3 & 7 & 0 & 4 & 6 & 107.65 & 0.55 \\
\hline CC13 & 4.906 & 426.822 & 3 & 5 & 0 & 4 & 5 & 61.83 & 0.55 \\
\hline CC14 & 4.728 & 406.403 & 3 & 5 & 0 & 4 & 5 & 61.83 & 0.55 \\
\hline CC15 & 4.99 & 471.273 & 3 & 5 & 0 & 4 & 5 & 61.83 & 0.55 \\
\hline
\end{tabular}

LogP - log of octanol/water partition coefficient; MW - molecular weight; nAR - nNumber of aromatic $\operatorname{ring}(\mathrm{s})$; nHBA - number of hydrogen bond acceptor(s); nHBD - number of hydrogen bond donor(s); nR - nNumber of ring(s); nRotB - number of rotable bond(s); Mol. PSA - molecular polar surface area

Table 4. Predicted ADMET properties of the designed 1,2,4-trioxane derivatives CC1-CC15

\begin{tabular}{c|c|c|c|c|c|c}
\hline $\begin{array}{c}\text { Compounds } \\
\text { code }\end{array}$ & AS & BBB & CYP P450 2D6 & HEPTOX & IA & PPB \\
\hline CC1 & 1 & 1 & true & true & 0 & true \\
\hline CC2 & 1 & 1 & true & true & 0 & true \\
\hline $\mathrm{CC} 3$ & 1 & 4 & true & true & 1 & true \\
\hline $\mathrm{CC} 4$ & 1 & 1 & false & true & 1 & true \\
\hline $\mathrm{CC} 5$ & 2 & 4 & true & true & 1 & true \\
\hline $\mathrm{CC} 6$ & 1 & 4 & true & true & 1 & true \\
\hline $\mathrm{CC} 7$ & 1 & 4 & false & true & 2 & true \\
\hline $\mathrm{CC} 8$ & 2 & 4 & true & true & 1 & true \\
\hline $\mathrm{CC} 9$ & 1 & 1 & false & true & 1 & true \\
\hline $\mathrm{CC} 10$ & 1 & 4 & true & true & 1 & true \\
\hline $\mathrm{CC} 11$ & 1 & 4 & true & true & 1 & true \\
\hline $\mathrm{CC} 12$ & 1 & 4 & false & true & 2 & true \\
\hline $\mathrm{CC} 13$ & 1 & 4 & true & true & 1 & true \\
\hline $\mathrm{CC} 14$ & 1 & 1 & false & true & 1 & true \\
\hline $\mathrm{CC} 15$ & 1 & 4 & true & true & 1 & true \\
\hline
\end{tabular}

AS (aqueous solubility) level: 2 - low, 1 - very low; BBB (blood-brain barrier) penetration: 4 - very low, 1 - high; CYP (cytochrome) P450 2D6 inhibition: true - inhibitor, false - noninhibitor; HEPTOX (hepatotoxicity): true - toxic; IA (intestinal absorption) level: 0 - good, 1 - moderate, 2 - high; PPB (plasma protein binding): true - highly bound, false - poorly bound 


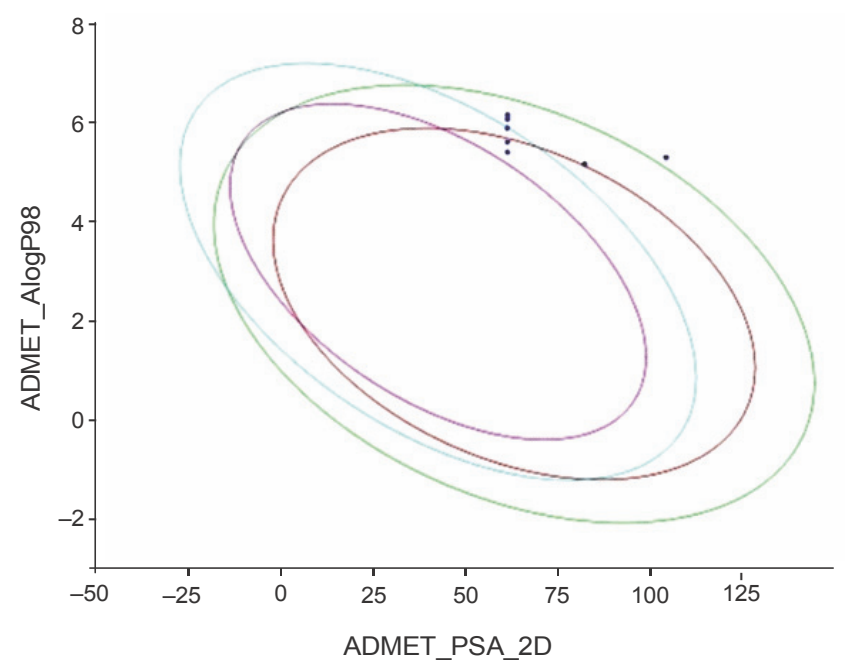

Fig. 5. ADMET plot of the designed 1,2,4-trioxane derivatives $\mathrm{CC} 1$ to $\mathrm{CC} 15$; the figure indicates that all the test compounds have acceptable ADMET characteristics; different colored lines represent different ADMET properties, while blue dots represent the test compounds with ADMET properties within the specified range

hibit predictable binding affinity against the $P$. falciparum cysteine protease. The 3D poses of protein-ligand complexes revealed predictive binding affinity of compounds relative to the molecular orientation of receptor molecule(s). Further analysis of the 2D interaction diagram indicated that polar hydrogen bonding interactions were primarily involved between the receptor and ligand molecule, along with some secondary interactions such as hydrophobic interactions. Well-defined molecular interactions between the binding site residues of receptor molecule(s) and complementary moieties/atoms of ligands were observed. The higher the number of hydrogen bonds, the higher was the binding affinity. Other nonbonded interactions such as hydrophobic bonding were also observed, but to a lesser extent. Four potent compounds, namely $\mathrm{CC} 3, \mathrm{CC} 5, \mathrm{CC} 7$, and $\mathrm{CC} 8$, interacted with different active site residues such as Gln 36, Ser 41, Cys 42, Trp 43, Gln 171, His 174, Trp 206, Asp 234, and Trp 43 predominantly by hydrogen bond formation. These compounds possess a substituted aromatic aldehyde with $-\mathrm{Br}$ group at position 4 , $-\mathrm{OH}$ group at position $3,-\mathrm{NO}_{2}$ at position 4 , and $-\mathrm{OH}$ at position 4. The compounds $\mathrm{CC} 3$ and $\mathrm{CC} 7$ exhibited well-defined protein-ligand interactions with very good binding modes with the formation of 7 and 5 hydrogen bonds with the FP- 2 protein molecule, respectively. The co-crystal ligand formed 11 hydrogen bonds. The details of 3D binding modes and 2D interaction diagrams of four active compounds and the co-crystal inhibitor E64 are shown in Fig. 4A-E.

\section{Drug-likeness}

The results of the calculated molecular properties and the predicted Lipinski's parameters are shown in Table 3. The results revealed that all the compounds showed good drug-like properties based on Lipinski's rule of five with additional parameters of drug-likeness. In our study, all the compounds exhibited satisfactory molecular properties and Lipinski's parameters. The parameters LogP, MW, and molecular PSA indicate good membrane permeability, intestinal absorption, and oral bioavailability, respectively, whereas the other parameters nHBAs, nHBDs, nR, and Rotb bonds facilitate to produce well-defined drug-receptor interactions for optimal drug action (Rudrapal et al., 2017). None of the compounds violated the Lipinski's rule of 5 . Drug-likeness scores were recorded as 0.55 for all the designed compounds. The drug-likeness scores were within the acceptable range $(0-1)$.

\section{ADMET}

The predicted ADMET data are presented in Table 4. All the compounds (CC1-CC15) showed low to very low aqueous solubility and exhibited very poor blood-brain barrier (BBB) penetration, thereby indicating less probability of inducing CNS toxicity. The compounds were found to be both inhibitors (CC1-CC3, CC5, CC6, CC8, CC10, CC11, CC12, CC13, and CC15) and noninhibitors (CC4, CC7, CC9, CC12, and $\mathrm{CC} 14)$ of cytochrome $\mathrm{P}_{450}$ 2D6 (CYP 2D6). The CYP 2D6 enzyme is one of the important metabolic enzymes involved in drug metabolism (Rudrapal et al., 2016b; Wang et al., 2009). The predictive hepatotoxicity was considerably observed for all the compounds. The intestinal absorption levels were observed in the range from good to very good. Plasma protein binding (PPB) data revealed that all the compounds were highly protein bound. The ADMET plot is shown in Fig. 5.

\section{Toxicity}

The toxicity data shown in Table 5A and Table 5B revealed that all the designed compounds were noncarcinogenic and nonmutagenic, except for a few ones (CC9, CC11, CC12, CC14, and CC15). Compounds CC3 and 
Table 5A. TOPKAT toxicity data of the designed 1,2,4-trioxane derivatives CC1-CC15

\begin{tabular}{l|c|c|c|c|c}
\hline $\begin{array}{c}\text { Compounds } \\
\text { code }\end{array}$ & $\begin{array}{c}\text { Mouse female } \\
\text { NTP }\end{array}$ & $\begin{array}{c}\text { Mouse male } \\
\text { NTP }\end{array}$ & $\begin{array}{c}\text { Rat female } \\
\text { NTP }\end{array}$ & $\begin{array}{c}\text { Rat male } \\
\text { NTP }\end{array}$ & $\begin{array}{c}\text { Rat oral LD } \\
{[\mathrm{g} / \mathrm{kg} \text { bw] }}\end{array}$ \\
\hline CC1 & carcinogen & carcinogen & non-carcinogen & non-carcinogen & 0.137904 \\
\hline CC2 & carcinogen & carcinogen & non-carcinogen & non-carcinogen & 0.173298 \\
\hline CC3 & non-carcinogen & carcinogen & non-carcinogen & non-carcinogen & 0.205028 \\
\hline CC4 & non-carcinogen & carcinogen & non-carcinogen & non-carcinogen & 0.330472 \\
\hline CC5 & non-carcinogen & non-carcinogen & non-carcinogen & non-carcinogen & 0.104876 \\
\hline CC6 & carcinogen & carcinogen & non-carcinogen & non-carcinogen & 0.101484 \\
\hline CC7 & non-carcinogen & non-carcinogen & non-carcinogen & non-carcinogen & 0.126426 \\
\hline CC8 & non-carcinogen & carcinogen & non-carcinogen & non-carcinogen & 0.104876 \\
\hline CC9 & non-carcinogen & carcinogen & non-carcinogen & carcinogen & 0.459507 \\
\hline CC10 & carcinogen & carcinogen & non-carcinogen & non-carcinogen & 0.119647 \\
\hline CC11 & carcinogen & carcinogen & non-carcinogen & carcinogen & 0.241720 \\
\hline CC12 & non-carcinogen & carcinogen & carcinogen & carcinogen & 0.126426 \\
\hline CC13 & carcinogen & carcinogen & non-carcinogen & non-carcinogen & 0.168420 \\
\hline CC14 & carcinogen & carcinogen & non-carcinogen & carcinogen & 0.102687 \\
\hline CC15 & carcinogen & carcinogen & non-carcinogen & carcinogen & 0.233167 \\
\hline
\end{tabular}

TOPKAT - Toxicity Prediction by Komputer Assisted Technology;

NTP - National Toxicology Program; LD - Lethal dose; bw - body weight

Table 5B. TOPKAT toxicity data of the designed 1,2,4-trioxane derivatives CC1-CC15

\begin{tabular}{|c|c|c|c|c|c|}
\hline $\begin{array}{l}\text { Compounds } \\
\text { code }\end{array}$ & $\begin{array}{c}\text { Mouse female } \\
\text { FDA }\end{array}$ & $\begin{array}{l}\text { Mouse male } \\
\text { FDA }\end{array}$ & $\begin{array}{c}\text { Rat female } \\
\text { FDA }\end{array}$ & $\begin{array}{c}\text { Rat male } \\
\text { FDA }\end{array}$ & $\begin{array}{c}\text { Ames } \\
\text { prediction }\end{array}$ \\
\hline $\mathrm{CC} 1$ & non-carcinogen & non-carcinogen & non-carcinogen & non-carcinogen & non-mutagen \\
\hline $\mathrm{CC} 2$ & non-carcinogen & non-carcinogen & non-carcinogen & non-carcinogen & non-mutagen \\
\hline $\mathrm{CC} 3$ & non-carcinogen & non-carcinogen & non-carcinogen & non-carcinogen & non-mutagen \\
\hline $\mathrm{CC} 4$ & non-carcinogen & non-carcinogen & non-carcinogen & non-carcinogen & non-mutagen \\
\hline $\mathrm{CC} 5$ & non-carcinogen & non-carcinogen & non-carcinogen & non-carcinogen & non-mutagen \\
\hline $\mathrm{CC} 6$ & non-carcinogen & non-carcinogen & non-carcinogen & non-carcinogen & non-mutagen \\
\hline $\mathrm{CC} 7$ & non-carcinogen & non-carcinogen & non-carcinogen & non-carcinogen & non-mutagen \\
\hline $\mathrm{CC} 8$ & non-carcinogen & non-carcinogen & non-carcinogen & non-carcinogen & non-mutagen \\
\hline $\mathrm{CC} 9$ & non-carcinogen & non-carcinogen & non-carcinogen & non-carcinogen & non-mutagen \\
\hline CC10 & non-carcinogen & non-carcinogen & non-carcinogen & non-carcinogen & non-mutagen \\
\hline CC11 & non-carcinogen & non-carcinogen & non-carcinogen & non-carcinogen & non-mutagen \\
\hline $\mathrm{CC} 12$ & non-carcinogen & non-carcinogen & non-carcinogen & non-carcinogen & mutagen \\
\hline CC13 & non-carcinogen & non-carcinogen & non-carcinogen & non-carcinogen & non-mutagen \\
\hline $\mathrm{CC} 14$ & non-carcinogen & non-carcinogen & non-carcinogen & non-carcinogen & non-mutagen \\
\hline CC15 & non-carcinogen & non-carcinogen & non-carcinogen & non-carcinogen & non-mutagen \\
\hline
\end{tabular}

FDA - food and drug administration; DTP - developmental toxicity potential 
A
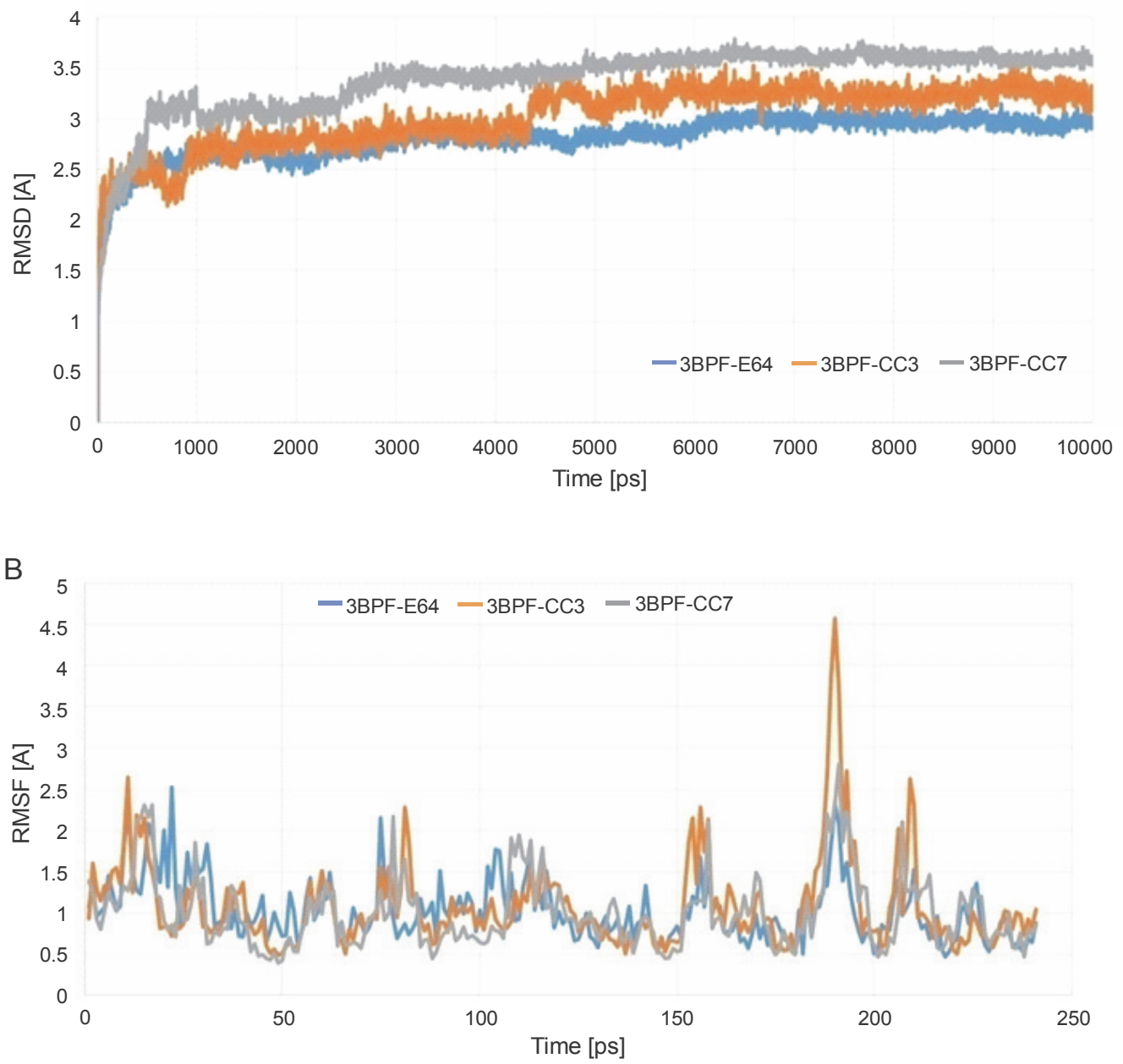

C

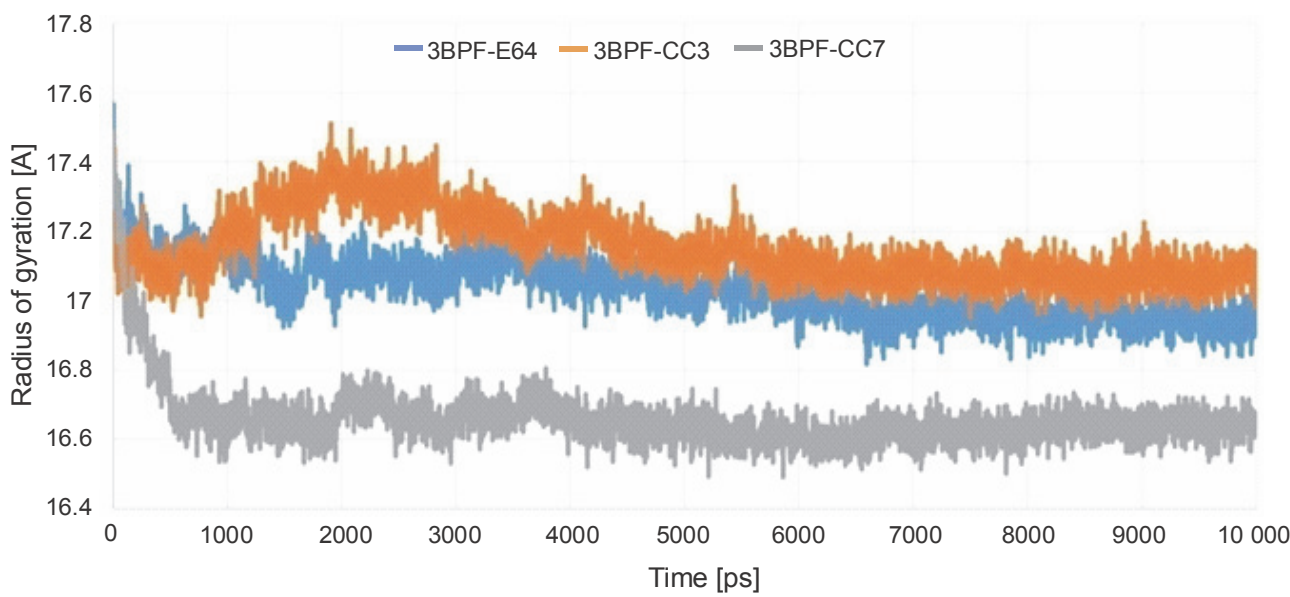

Fig. 6. Stability indicating parameters obtained from the MD simulation analysis A) RMSD values,

B) RMSF values, and C) ROG values; the blue line indicates the 3BPF-E64 complex, the orange line indicates the 3BPF-CC 3 complex, and the gray line indicates the 3BPF-CC7 complex

$\mathrm{CC} 7$ were found to be noncarcinogenic and nonmutagenic. They were found to be safe and nontoxic in various in silico models of nonruminant animals.

\section{MD simulation}

After completing the MD simulation of $10 \mathrm{~ns}$, it was observed that the protein-ligand complexes formed by 
A

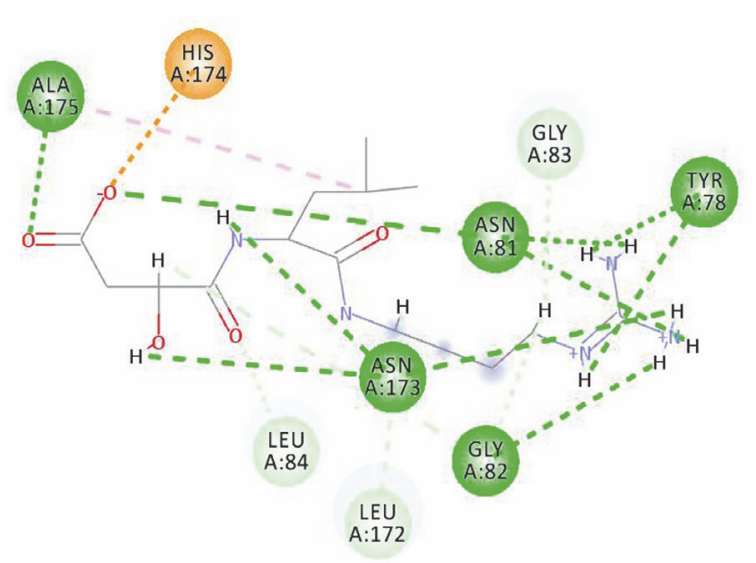

B

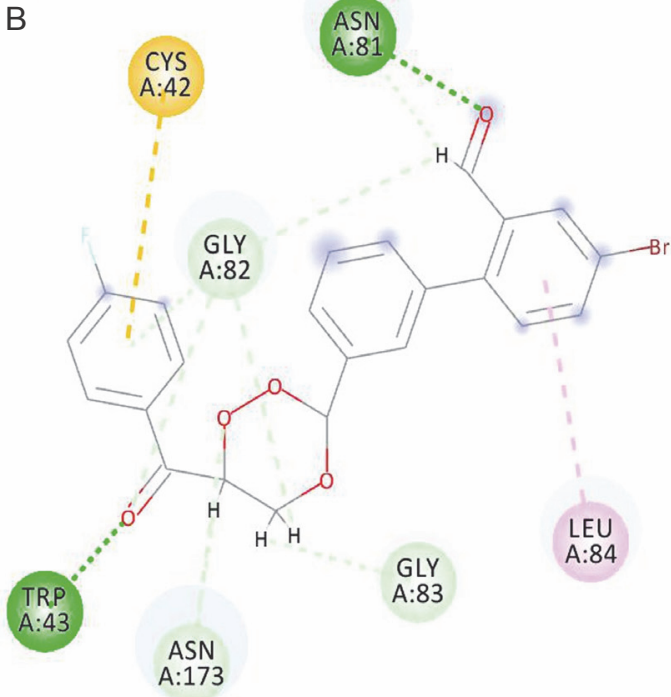

C

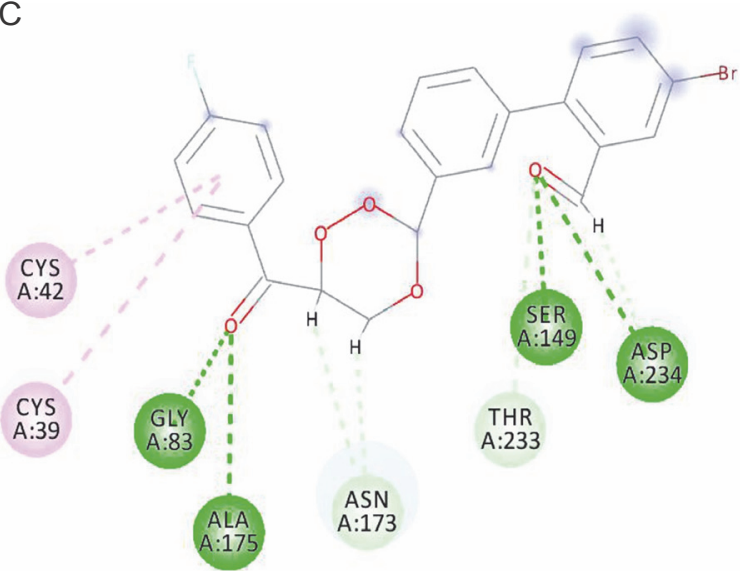

Fig. 7. Interactions of the compounds with the amino acid residues of the active binding site of 3BPF: A) E64 with 3BPF; B) CC3 with $3 \mathrm{BPF}$, and $\mathrm{C}$ ) $\mathrm{CC} 7$ with $3 \mathrm{BPF}$; three green dash lines represent the hydrogen bonds formed by the compounds

both the compounds $\mathrm{CC} 3$ and $\mathrm{CC} 7$ with the co-crystal ligand E64 reached the plateau states within $1 \mathrm{~ns}$ (Fig. 10). Within the $10 \mathrm{~ns}$ of simulation, the 3BPF-E64 complex deviated less than the complexes formed by the other two test compounds (CC3 and CC7), but the differences between their deviations were very small and nonsignificant (Fig. 6). The ROG diagram indicated that the 3BPF-E64 and 3BPF-CC3 complexes were formed with almost equal compactness, but $\mathrm{CC} 7$ formed a more compact and tighter complex with FP-2 (3BPF) than the other two complexes (Fig. 6).

The interactions of the average protein-ligand complexes obtained after the MD simulation study showed that the co-crystal ligand formed 13 hydrogen bonds with the protein $3 \mathrm{BPF}$, whereas $\mathrm{CC} 3$ formed only two and
$\mathrm{CC} 7$ formed only four hydrogen bonds with 3BPF. Other than the hydrogen bonds, all the compounds formed some hydrophobic interactions (Fig. 7) in the active binding site of 3BPF. Amino acid residues such as Trp 43, Asn 81, Gly 82, Gly83, Leu 84, Ser 149, Asn 173, Ala 175, Thr 233, and Asp 234 were involved in the interactions with trioxane molecules. Later, the fluctuations of the distances of hydrogen bonds formed were calculated, and we found that for E64, all the hydrogen bonds were stabilized within $2 \mathrm{~ns}$ and the distances were maintained under $5 \AA$ after $2 \mathrm{~ns}$. Even for CC3, the hydrogen bonds were stabilized within $2 \mathrm{~ns}$, and the distances were maintained under $5 \AA$ after $2 \mathrm{~ns}$ of the simulation period. For $\mathrm{CC} 7$, the hydrogen bonds were highly fluctuated within $3 \mathrm{~ns}$, but subsequently, they were stabilized and fluctu- 


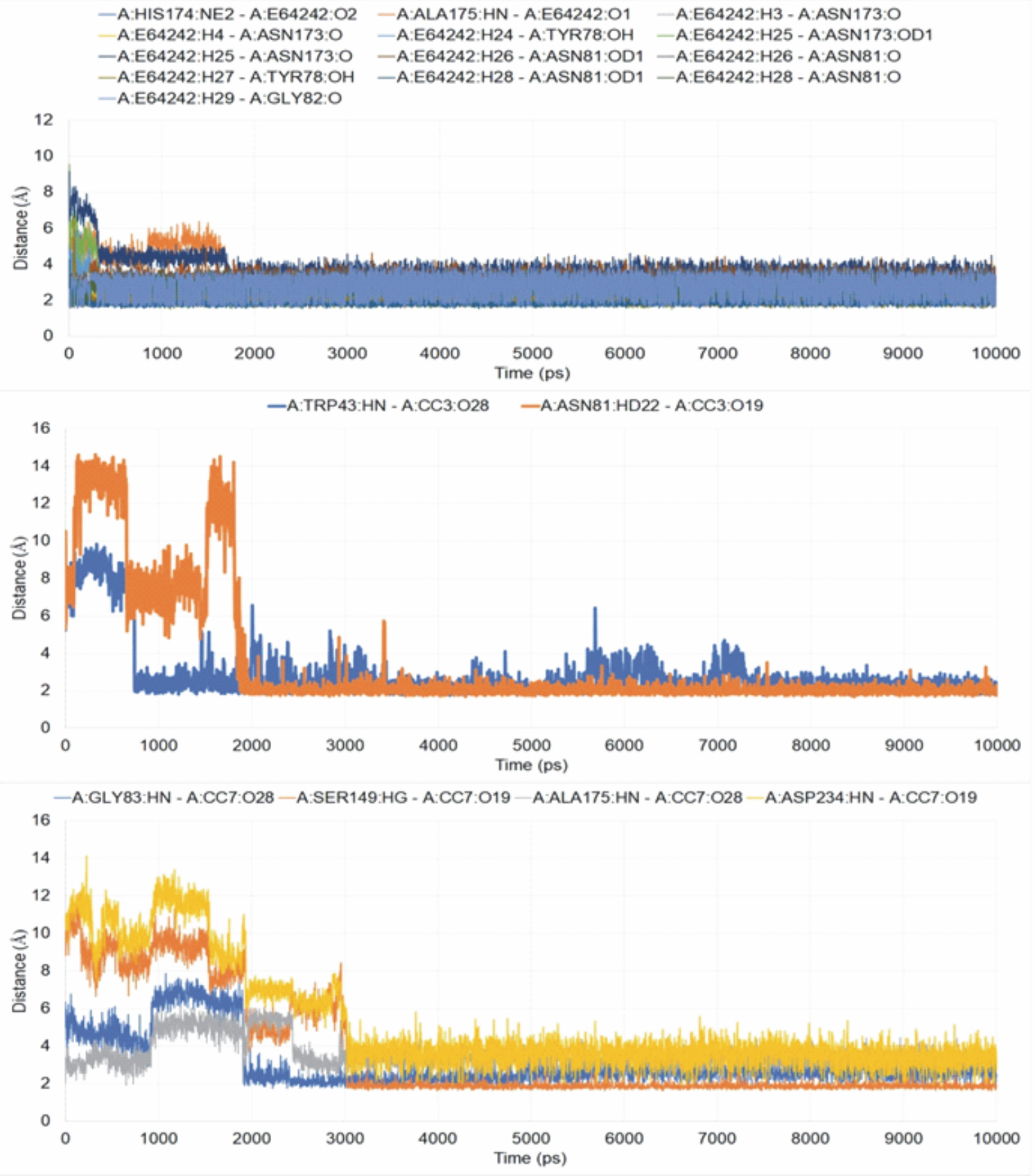

Fig. 8. Fluctuations of the distances of the hydrogen bonds within the simulation period for A) 3BPF-E64, B) 3BPF-CC3, and C) 3BPF-CC7 complexes

ated under $5 \AA$ until the end of the simulation period (Fig. 8).

\section{MMPBSA-based binding free energy}

After completing the MD simulation, the MM-PBSAbased binding free energies were calculated for all the generated conformations, and the average binding free energy was then calculated for each protein-ligand complex (Fig. 9). The binding free energy of the test compounds (CC3: $-55.078 \mathrm{kcal} / \mathrm{mol}$ and CC7:
$-62.241 \mathrm{kcal} / \mathrm{mol}$ ) were found to be considerably higher than that of the co-crystal ligand E64 $(-33.079 \mathrm{kcal} / \mathrm{mol})$. This indicated that the test compounds ( $\mathrm{CC} 3$ and $\mathrm{CC} 7$ ) formed thermodynamically stable complexes with the target protein, which are comparable to that of the complex formed with the co-crystal ligand E64.

\section{Docking for selectivity study}

Table 6 presents the results of docking (LibDock scores) for the selectivity study of compounds CC3 and 


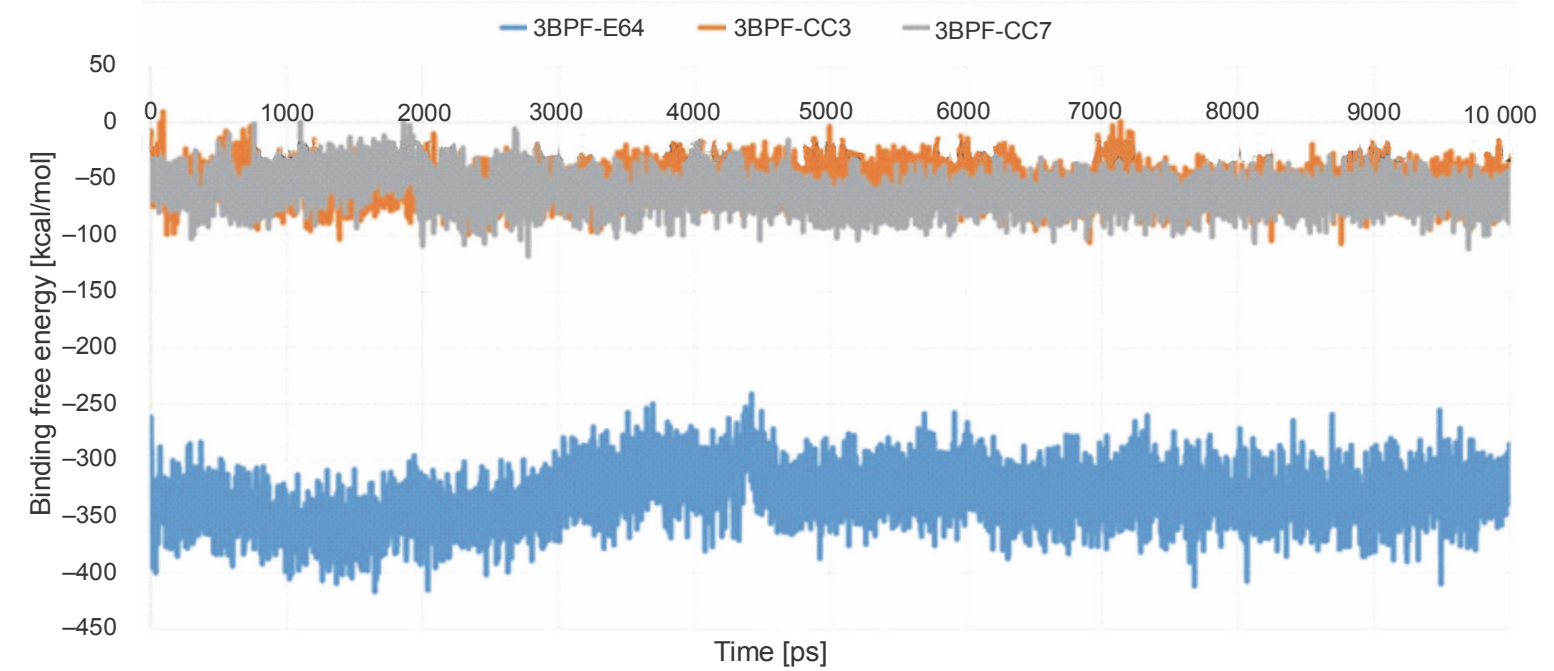

Fig. 9. Binding free energies of the conformations generated within $10 \mathrm{~ns}$; the blue line represents the 3BPF-E64 complex, the orange line represents the 3BPF-CC3 complex, and the gray line represents the 3BPF-CC7 complex

Table 6. LibDock scores of 1,2,4-trioxane derivatives CC3 and $\mathrm{CC} 7$ against cathepsin $\mathrm{K}$ and cathepsin $\mathrm{L}$

\begin{tabular}{l|c|c|c|c}
\hline \multirow{2}{*}{ Compounds code } & \multicolumn{2}{|c|}{ LibDock score } & \multicolumn{2}{c}{ Number of H-bonds } \\
\cline { 2 - 5 } & 3 OVZ & 3OF9 & 3OVZ & 3OF9 \\
\hline CC3 & 140.913 & 120.04 & 2 & 2 \\
\hline CC7 & 137.396 & 130.701 & 2 & 3 \\
\hline Co-crystal inhibitor & 134.625 & 152.045 & 6 & 5 \\
\hline
\end{tabular}

$30 \mathrm{VZ}$ - cathepsin K (co-crystal inhibitor: 096); $30 F 9$ - cathepsin L (co-crystal inhibitor: IOX)

$\mathrm{CC} 7$ against $\mathrm{Cat} \mathrm{K}$ and $\mathrm{Cat} \mathrm{L}$. Both $\mathrm{CC} 3$ and $\mathrm{CC} 7 \mathrm{ex}-$ hibited selectivity against both the host proteins in terms of LibDock scores. Compound CC3 showed higher selectivity on Cat K with the LibDock score of 140.913, whereas compound $\mathrm{CC} 7$ exhibited higher selectivity on Cat $\mathrm{L}$ with the LibDock score of 130.701 . Selectivity of trioxane compounds ( $\mathrm{CC} 3$ and $\mathrm{CC} 7$ ) was comparable to that of the co-crystal ligands (O96 and I0X). Figure 10 and Figure 11 shows binding modes and interactions (2D and 3D) of docking for compounds CC3 and CC7 against the host homologous cysteine proteases Cat $\mathrm{K}$ and Cat L, respectively. For human proteases, the interactions between protein amino acid residues and ligand atoms were observed with the formation of hydrogen and hydrophobic bonds. For Cat K, two H-bonds were formed with both the compounds $\mathrm{CC} 3$ and $\mathrm{CC} 7$, while, for Cat L, two and three H-bonds were observed for compounds $\mathrm{CC} 3$ and $\mathrm{CC} 7$, respectively. The co-crystal ligand formed six and five $\mathrm{H}$-bonds for Cat $\mathrm{K}$ and $\mathrm{Cat} \mathrm{L}$, respectively. Amino acid residues such as Gln 21, Gly 23, Gly 69, His 164, Ala 163, and Gly 165 of Cat K and $\mathrm{Cat} \mathrm{L}$ proteins were involved in $\mathrm{H}$-bond formation with the ligand molecules CC3 and CC7.

\section{Discussion}

In our study, 15 new 1,2,4-trioxane derivatives were designed by the molecular manipulation approach of rational drug design (RDD) and reported as novel FP-2 inhibitors with predictive (in silico) antimalarial potential against $P$. falciparum. In ART-based antimalarial drugs, 1,2,4-trioxane is believed to be the key pharmacophoric moiety responsible for their antiparasitic effect (Rudrapal et al., 2017). In view of this fact, novel 1,2,4trioxane derivatives were designed by structural modification of 1,2,4-trioxane pharmacophoric skeleton with the incorporation of substituted bulky aryl moieties. Rudrapal et al. (2017) designed a series of 1,2,4-trioxane 


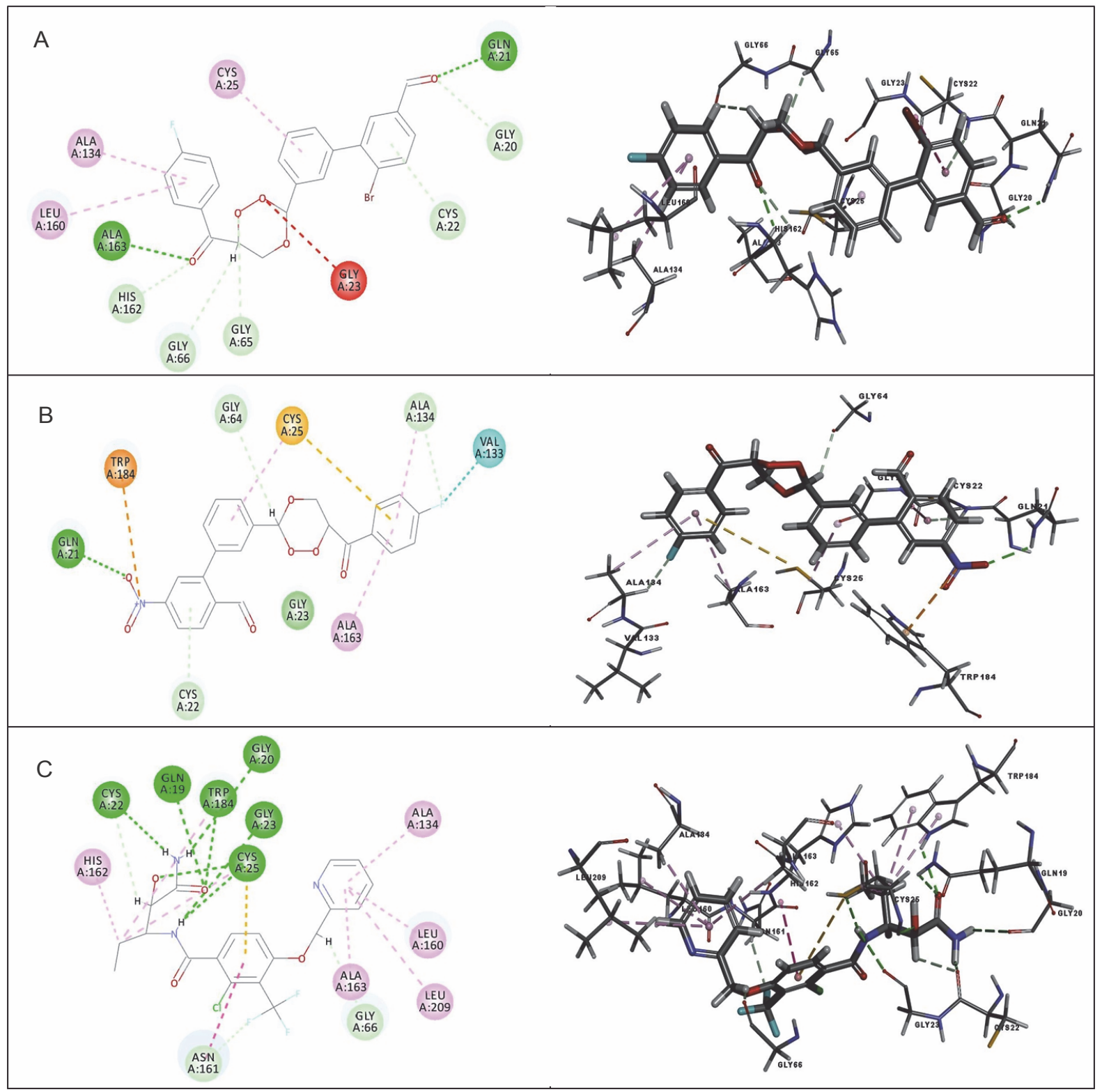

Fig. 10. Binding modes and docking interactions diagrams of compounds CC3 (A), CC7 (B), and the co-crystal ligand O96 (C) (left: 2D interaction, right: 3D interaction) against cathepsin $\mathrm{K}(3 \mathrm{OVZ})$; dotted green lines indicate conventional H-bonding interactions, and dotted yellow and purple lines indicate different types of hydrophobic interactions

derivatives with different alkyl/aryl/heteroaryl substitutions at the $\mathrm{C}-3$ position of the 1,2,4-trioxane scaffold. They reported that the presence of aryl/heteroaryl moieties could greatly modulate the antimalarial efficacy of the basic trioxane scaffold. Therefore, no alternations were made in the parent structural framework of 1,2,4-trioxane skeleton which is an essential requirement for the antimalarial activity, but a structural modification was made by incorporating biologically/pharmacokinetically relevant bulky aryl substituents (4-fluoro- benzoyl and substituted biphenyl-2-carbaldehyde). Molecular docking is a virtual tool intended to find the best binding orientation of small molecules bound to their target protein molecules. It is used to predict the binding affinity and biological efficacy of small molecules (Ghorab et al., 2020; Abdel-Hamid et al., 2014). Moreover, docking plays an important role in the identification of bioactive molecules based on the target protein structure in RDD and discovery program (Rudrapal et al., 2018). The docking protocol was validated by re- 


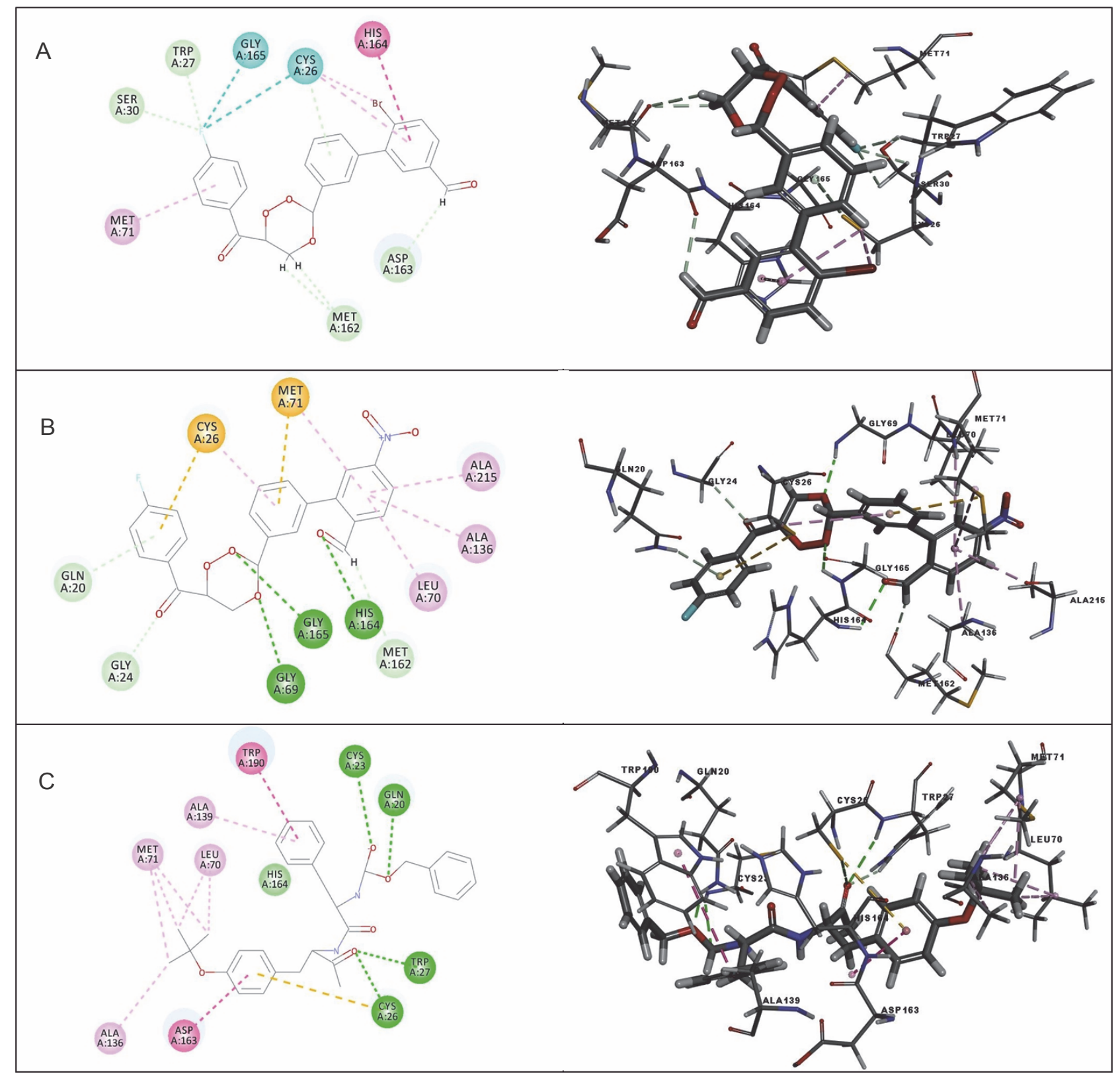

Fig. 11. Binding modes and docking interaction diagrams of compounds CC3 (A), CC7 (B), and the co-crystal ligand I0X (C) (left: $2 \mathrm{D}$ interaction, right: $3 \mathrm{D}$ interaction) against cathepsin $\mathrm{K}(3 \mathrm{OF} 9)$; dotted green lines indicate conventional H-bonding interactions, and dotted yellow and purple lines indicate different types of hydrophobic interactions

docking the co-crystal ligand into the predicted binding sites of the target receptor protein followed by analyzing the binding modes of the test compound in the docked complex by comparing with the binding modes of the cocrystal inhibitor E64 (Rudrapal et al., 2019). The cocrystallized ligand was re-docked using flexible docking simulations into the original structure of the receptor molecule by setting all the docking parameters to the software's default values. The validation of the docking method confirmed the accuracy of the procedure with an acceptable RMSD of less than $2 \AA$. The results of our docking study are consistent with previous results reported in the literature. Kalita et al. (2019) recorded similar observations while performing validation of the docking method using the FP-2 protein (3BPF) in their study. This further substantiates the validation of the docking method reported in our study.

The protein-ligand docking was performed to predict the binding affinity of the test compounds $\mathrm{CC} 1-\mathrm{CC} 15$ as possible novel antimalarial agents with inhibitory activity 
against $P$. falciparum FP-2. FP-2 is a cysteine protease enzyme that plays an important role during the process of hemoglobin degradation in the food vacuole of $P$. falciparum during the erythrocytic life cycle of the parasite (Rudrapal et al., 2017; Grazioso et al., 2012). In the protein-ligand docking study, the LibDock program successfully docked all the compounds $\mathrm{CC} 1-\mathrm{CC} 15$ into the binding pocket of protein molecules. The compounds could bind well with the predefined active site residues of the predicted receptor spheres depicted in the receptor grid model. Higher binding affinities in terms of LibDock scores were observed for all the deigned compounds as compared to the co-crystal inhibitor E64. In the proteinligand interaction analysis, it was found that the basic 1,2,4-trioxane moiety interacted with the amino acid residues (such as Gln 36, Ser 41, Cys 42, and His 174 depicted in the results section) at the active site predominantly through the formation of H-bonds, and aryl rings and substituent groups such as $-\mathrm{OH},-\mathrm{CH}_{3}$, and $-\mathrm{NO}_{2}$ were also involved in interaction by $\mathrm{H}$-bonding and various hydrophobic interactions such as Van der Waals and pi-alkyl bonds. A strong interaction of the trioxane pharmacophore with the predicted binding sites of FP-2 was observed. Substituents such as $-\mathrm{OH}$ and $-\mathrm{CH}_{3}$ further modulated the strength of interaction to achieve optimal interaction. In a previous study, Rudrapal et al. (2018) reported that 1,2,4-trioxane interacted with the FP-2 protein through the formation of hydrogen and hydrophobic bonds. In their study, besides the trioxane pharmacophore, various substituents/moieties also contributed to the molecular interactions between the key pharmacophoric trioxane structure and various amino acid residues of the FP-2 protein. According to their reports, the trioxane pharmacophore significantly interacted with certain key amino acid residues such as Cys 42, Gly 83, and His 174, with the LibDock score ranging from 93 to 126 against FP-2. Although our present study is consistent with previous findings, the trioxane derivatives reported here possess greater FP-2 inhibitory potential (in terms of LibDock score and H-bonding interactions) than trioxanes reported by Rudrapal et al. (2017). The present trioxane derivatives exhibited comparatively higher LibDock scores $(>100)$ with the formation of more number of $\mathrm{H}$-bonds. This clearly reflects the stronger binding affinity/inhibitory potential against the FP-2 protein. From receptor-ligand interactions, it is also evident that the substituted aryl moieties $\left(\mathrm{HC}=\mathrm{O}, \mathrm{C}=\mathrm{O}\right.$, and $\left.\mathrm{NO}_{2}\right)$ further modulate the binding affinity besides the direct inhibitory role of the trioxane scaffold against FP-2. Furthermore, literature also confirm that the inhibition of the FP-2 protein by structural scaffolds (such as 1,4-benzodiazepine) other than trioxane is due to the involvement of the interacting amino acid residues such as Cys 42 and His 174 (Giovanni et al., 2012). These observations further strengthen our findings. Considering all these, it is apparent that the 1,2,4-trioxane scaffold- based derivatives designed in our study could offer a novel pharmacophoric moiety for further modification toward the development of new trioxane-based compounds/agents as promising $P$. falciparum FP-2 inhibitors with in vitro/ in vivo antimalarial activities. However, subtle structural modifications must be performed to improve the antimalarial potency and to avoid undesired toxic effects such as hepatotoxicity and mutagenic properties.

In our study, all compounds obeyed "Lipinski's rule of five" and "Veber rule." According to Lipinski's rule, compounds are more likely to be drug-like and orally bioavailable if they obey the following criteria: $\log \mathrm{P}_{\mathrm{o} / \mathrm{w}}$ (octanol/water partition coefficient) $\leq 5, \mathrm{MW} \leq 500$, nHBAs $\leq 10$, and nHBDs $\leq 5$ (Jain et al., 2008). To further substantiate drug-likeness, Veber et al. (2002) stated that compounds with $\leq 10$ RotB and molecular PSA of $\leq 140 \mathrm{~A}^{2}$ are more likely to show optimum membrane permeability and good bioavailability. In ADMET prediction, aqueous solubility, BBB penetration, CYP P450 2D6 inhibition, hepatotoxicity, intestinal absorption, and plasma protein binding were in acceptable limits (Rudrapal et al., 2017; Ponnan et al., 2013; Rudrapal et al., $2016 \mathrm{~b})$. The predicted toxicity data such as $\mathrm{LD}_{50}$ in mice and rats, Ames mutagenicity, DTP, and skin irritancy did not cross the limit of toxicity. Previous studies support the results of our present studies related to the prediction of toxicity parameters of trioxane derivatives (Rudrapal et al., 2019). Thus, it can be stated that the designed trioxane derivatives possess drug-likeness and ADMET properties within the acceptable range and do not have any predictive toxic features.

MD simulation study, which provides more accurate and reliable information than simple molecular docking study, was performed to observe the stability of the protein-ligand complexes. In the MD simulation analysis, both protein and ligand structures remain flexible, thus allowing for an induced fit into the binding site of the 
receptor around the introduced ligand (Lin et al., 2011). MD simulation was analyzed on the basis of RMSD, RMSF, and ROG as a function of time. Fluctuations of the distances of the hydrogen bonds within the simulation period of $10 \mathrm{~ns}$ were determined. The MM-PBSA method is a popular approach to estimate the free energy of the binding of small ligands to proteins. These methods are typically based on MD simulations of the receptor-ligand complex and are therefore intermediate in both accuracy and computational effort between empirical scoring and strict alchemical perturbation methods (Rastelli et al., 2010).The MM-PBSA-based calculation of binding free energy $(\Delta G)$ is one of the important parameters to estimate the binding affinity of a compound to a protein target as well as the thermodynamic stability of the protein-ligand complex (Genheden and Ryde, 2015). This technique provides a fast and accurate prediction of absolute binding affinity of a compound within the active binding site of a target protein in the form of binding free energy, which is very important for stability and particular potency of the compound (Rastelli et al., 2010). Analysis of the results of MD simulation revealed that the trioxane compounds formed stale complexes with the Plasmodium cysteine protease FP-2. The fluctuations of the amino acid residues in all the three cases of RMSD, RMSF, and ROG determinations were very small, and no significant differences were observed. The MD simulation study thus confirms the predictivity of binding affinity of the trioxane compounds with the FP-2 molecule. Furthermore, the analysis of MM-PBSA-based binding free energies revealed the formation of thermodynamically stable (during the simulation period of $10 \mathrm{~ns}$ ) complexes between trioxanes and the FP-2 molecule. From the above observations, it is apparent that the trioxane pharmacophore along with the structural substituents contributed significantly to the formation of strong and stable protein-ligand complexes. Musyoka et al. (2016b) demonstrated well-defined and stable protein-ligand complexes where nonpeptidic compounds (chalcones and isoquinolenes) interacted with the involvement of some key amino acid residues (Asn, Cys, Gln, and His) from Plasmodium FP-2 protease, which substantiates the FP-2 inhibitory potential of trioxanes reported in this study.

Results of the selectivity study indicate that in addition to higher binding affinities against Plasmodium cysteine protease FP-2, the trioxane compounds showed lesser selectivity on human homologous proteases Cat $\mathrm{K}$ and Cat L. This implies that the trioxane compounds have more binding affinity for the parasitic protease (FP-2) over the host proteases (Cat K or Cat L). The enzymatic selectivity for the human proteases is not significant for the trioxane molecules. This study therefore proves that trioxane molecules are devoid of any interactions/toxic effects on humans. In the docking study against the FP-2 molecule, two best scored compounds CC3 and CC7 with well-defined H-bonding interactions with the FP-2 protein formed 7 and $5 \mathrm{H}$-bonds, respectively. In human Cat $\mathrm{K}$ and $\mathrm{Cat} \mathrm{L}$, the number of H-bonds between amino acids and ligands was comparatively lower to that of Plasmodium FP-2. The molecular interactions predicted in docking in terms of $\mathrm{H}$-bonding occupancy and bond strength were comparatively less significant for the human proteases cathepsins than for Plasmodium protease FP-2. Similar finding have been observed in recent studies reported in literature (Musyoka et al., 2016a; Musyoka et al., 2016b), which substantiates the results of our study. The authors reported marginal selectivity of their designed FP-2 inhibitors on human homologous cathepsins.

\section{Conclusions}

In the present study, 15 new trioxane derivatives were designed based on the 1,2,4-troxane pharmacophoric scaffold of artemisinin by the molecular modification approach. The designed compounds were investigated by molecular modeling studies, including molecular docking, drug-likeness, ADMET, and toxicity assessment and further MD simulation and MM-PBSA studies. Among the 15 trixoane derivatives, two compounds, namely CC3 and CC7, were found to exhibit the highest binding affinity against the $P$. falciparum cysteine protease FP-2 enzyme in terms of LibDock score. These compounds are therefore suggested to be potent molecules with FP-2 inhibitory activity. All compounds exhibited satisfactory in silico drug-likeness, ADMET, and toxicity properties. No compounds violated the Lipinski's rule of five, and the drug-likeness scores of all the compounds were within the acceptable range. MD studies further confirmed the antimalarial potential of the compounds $\mathrm{CC} 3$ and $\mathrm{CC} 7$ with the formation of welldefined and stable receptor-ligand interactions against the $P$. falciparum FP-2 enzyme. The MM-PBSA study 
indicated that compounds $\mathrm{CC} 3$ and $\mathrm{CC} 7$ formed thermodynamically stable complexes with the target protein FP-2, which are comparable to the complexes formed with the co-crystal ligand E64. Furthermore, in addition to the inhibitory activity of $\mathrm{CC} 3$ and $\mathrm{CC} 7$ against Plasmodium cysteine protease FP-2, the selectivity on human cathepsins (Cat $\mathrm{K}$ and Cat $\mathrm{L}$ ) was identified. It can be concluded that the newly designed 1,2,4-trioxane derivatives can be further investigated for in vitro and in vivo antimalarial activities for their possible development as potent antimalarial agents effective against resistant strains of $P$. falciparum.

\section{Acknowledgment}

The authors would like to thank Dr. Sagarika Chandra for her kind and generous help in editing the figures/images of the manuscript.

\section{References}

Abdel-Hamid M.K., McCluskey A. (2014) In silico docking, molecular dynamics and binding energy insights into the bolinaquinone-clathrin terminal domain binding site. Molecules 19: 6609-6622.

Biamonte M.A., Warner J., Le Roch K.G. (2013) Recent advances in malaria drug discovery. Bioorg. Med. Chem. Lett. 23: $2829-2843$.

Chakroborty S., Bhanja C., Jena S. (2012) Advances in the synthesis of biologically important 1,2,4-trioxanes. Asian J. Biomed. Pharm. Sci. 2: 1-7.

Cumming J.N., Wang D., Park S.B., Shapiro T.A., Posner G.H. (1998) Design, synthesis, derivatization, and structure-activity relationships of simplified, tricyclic, 1,2,4-trioxane alcohol analogues of the antimalarial artemisinin. J. Med. Chem. 41(6): 952-964.

Genheden S., Ryde U. (2015) The $M M / P B S A$ and $M M / G B S A$ methods to estimate ligand-binding affinities. Expert Opin. Drug Discov. 2015: 449-461.

Ghorab M.M., Soliman A.M., Alsaid M.S., Askar A.A. (2020) Synthesis, antimicrobial activity and docking study of some novel 4-(4,4-dimethyl-2,6-dioxocyclohexylidene)methylamino derivatives carrying biologically active sulfonamide moiety. Arabian J. Chem. 13: 545-556.

Gogoi J., Chetia D., Kumawat M.K., Rudrapal M. (2016) Synthesis and antimalarial activity evaluation of some mannich bases of tetraoxane-phenol conjugate. Indian J. Pharm. Edu. Res. 50(4): 591-597.

Grazioso G., Legnani L., Toma L., Ettari R., Micale N., De Micheli C. (2012) Mechanism of falcipain-2 inhibition by $\alpha, \beta$ unsaturated benzo $[1,4]$ diazepin-2-one methyl ester. J Comput. Aided Mol. Des. 26(9): 1035-1043.

Kashyap A., Chetia D., Rudrapal M. (2016) Synthesis, antimalarial activity evaluation and drug-likeness study of some new quinoline-lawsone hybrids. Indian J. Pharm. Sci. 78(6): 892-911.
Jain A.N.B. (2008) Bias, reporting and sharing: computational evaluations of docking methods. J. Comput. Aided Mol. Des. 22(3-4): 201-212.

Kalita J., Chetia D., Rudrapal M. (2020) Design, synthesis, antimalarial activity and docking study of 7-chloro-4-(2-(substituted benzylidene)hydrazineyl)quinolines. Med. Chem. 16: 928-937.

Kalita J., Chetia D., Rudrapal M. (2019) Molecular docking, drug-likeness studies and ADMET prediction of quinoline imines for antimalarial activity. J. Med. Chem. Drug Des. 2(1): 1-7.

Lai C.Y., Chang T.T., Sun M.F., Chen H.Y., Tsai F.J., Lin J.G., Chen C.Y.C. (2011) Molecular dynamics analysis of potent inhibitors of M2 proton channel against H1N1 swine influenza virus. Mol. Simul. 37: 250-256.

Lin C.H., Chang T.T., Sun M.F., Chen H.Y., Tsai F.J., Chang K.L., Fisher M., Chen C.Y.C. (2011) Potent inhibitor design against h1n1 swine influenza: Structure-based and molecular dynamics analysis for $\mathrm{m} 2$ inhibitors from traditional chinese medicine database. J. Biomol. Struct. Dyn. 28: 471-482.

Medhi A., Chetia D., Rudrapal M. (2018) Synthesis and antimalarial activity of LawsoneMannich base derivatives. Indian J. Pharm. Edu. Res. 52(3): 472-479.

Noha S.M., Schmidhammer H., Spetea M. (2017) Molecular docking, molecular dynamics, and structure-activity relationship explorations of 14-oxygenated $\mathrm{N}$-methylmorphinan-6-ones as potent $\mu$-opioid receptor agonists. ACS Chem. Neurosci. 8: 1327-1337.

Mugumbate G., Newton A.S. (2013) Novel anti-plasmodial hits identified by virtual screening of the ZINC database. J. Comput. Aided Mol. Des. 27: 859-871.

Musyoka T.M., Kanzi A.M., Lobb K.A., Bishop Ö.T. (2016a) Structure based docking and molecular dynamic studies of plasmodial cysteine proteases against a South African natural compound and its analogs. Sci. Rep. 6: 1-12.

Musyoka T.M., Kanzi A.M., Lobb K.A., Bishop Ö.T. (2016b) Analysis of non-peptidic compounds as potential malarial inhibitors against plasmodial cysteine proteases via integrated virtual screening workflow. J. Biomol. Struct. Dyn. 34: 2084-2101.

O'Neill P.M., Rawe S.L., Borstnik K., et al. (2005) Enantiomeric 1,2,4-trioxanes display equivalent in vitro antimalarial activity versus Plasmodium falciparum malaria parasites: implications for the molecular mechanism of action of the artemisinin. Chem. Bio. Chem. 6: 2048-2054.

Opensica D.M., Solaja B. (2009) Antimalarial peroxides. J. Serb. Chem. Soc. 74(11): 1155-1193.

Patowary P., Chetia D., Kalita J., Rudrapal M. (2019) Design, synthesis and antimalarial activity of flavonoid derivatives. Indian J. Heterocycl. Chem. 29(1): 53-58.

Ponnan P., Gupta S., Chopra M., Tandon R., Baghel A.S., Gupta G., Prasad A.K., Rastogi R.C., Bose M., Raj H.G. (2013) 2DQSAR, docking studies, and in silico ADMET prediction of polyphenolic acetates as substrates for protein acetyltransferase function of glutamine synthetase of Mycobacterium tuberculosis. ISRN Struct. Biol. 2013: 1-12. 
Posner G.H. (1997) Trioxane dimmers have potent antimalarial, antiproliferative and antitumor activities. Bioorg. Med. Chem. 5(7): 1257-1265.

Rastelli G., Del Rio A., Degliesposti G., Sgobba M. (2010) Fast and accurate predictions of binding free energies using MM-PBSA and MM-GBSA. J. Comput. Chem. 31: 797-810.

Robert A., Benoit-Vical F., Dechy-Cabaret O., Meunier B. (2001) From classical antimalarial drugs to new compounds based on the mechanism of action of artemisinin. Pure Appl Chem. 73(7): 1173-1180.

Roy S., Chetia D., Rudrapal M., Prakash A. (2013) Synthesis and antimalarial activity study of some new Mannich bases of 7-chloro-4-aminoquinoline. Med. Chem. 9(3): 379-383.

Rudrapal M., Banu Z.W., Chetia D. (2018) Newer series of trioxane derivatives as potent antimalarial agents. Med. Chem. Res. 27(2): 653-668.

Rudrapal M., Chetia D. (2016a) Endoperoxideantimalarials: development, structural diversity and pharmacodynamic aspects with reference to 1,2,4-trioxane-based structural scaffold. Drug Des. Devl. Ther. 10: 3575-3590.

Rudrapal M., Chetia D. (2016b) QSAR analysis of 7-chloro-4aminoquinoline derivatives as antimalarial agents. Asian J. Org. Med. Chem. 1(2): 51-54.

Rudrapal M., Chetia D., Singh V. (2017) Novel series of 1,2,4trioxane derivatives as antimalarial agents. J. Enzyme Inhib. Med. Chem. 32(1): 1159-1173.

Rudrapal M., Chetia D. (2017) Plant flavonoids as potential source of future antimalarial leads. Sys. Rev. Pharm. 8(1): 28-33.
Rudrapal M., Chetia D. (2019) In vitro and in vivo models used for antimalarial activity: a brief review. Asian J. Pharm. Pharmacol. 5(6): 1251-1255.

Rudrapal M., Chetia D., Prakash A. (2013) Synthesis, antimalarial and antibacterial activity evaluation of some new 4-aminoquinoline derivatives. Med. Chem. Res. 22(8): 3703-3711.

Rudrapal M., Mullapudi S. (2019) Design, synthesis, drug-likeness studies and bio-evaluation of some novel chalconeimines. Pharm. Chem. J. 53(9): 814-821.

Sharma D., Chetia D., Rudrapal M. (2016) Design, aynthesis and antimalarial activity of new 2-hydroxy-1,4-naphthoquinone-4-hydroxyanilino hybrid Mannich bases. Asian J. Chem. 28(4): 782-788.

Veber D.F., Johnson S.R., Cheng H.Y., Smith B.R., Ward K.W., Kopple K.D. (2002) Molecular properties that influence the oral bioavailability of drug candidates. J. Med. Chem. 45(12): 2615-2623.

Wang X., Greek D.J., Dong Y. (2009) Spiroadamantyl 1,2,4trioxolane, 1,2,4-trioxane, and 1,2,4-trioxepane pairs: relationship between peroxide bond iron(II) reactivity, haem alkylation efficiency, and antimalarial activity. Bioorg. Med. Chem. Lett. 19(16): 4542-4545. 